\title{
Bioorganic chemistry of rhodopsins
}

\author{
Yuri A. Ovchinnikov \\ Shemyakin Institute of Bioorganic Chemistry, USSR Academy of Sciences, \\ 117981 Moscow V-437, USSR
}

\begin{abstract}
Bacteriorhodopsin and rhodopsin are integral membrane proteins containing retinal as a light sensitive group. Light energy is used by halophilic microorganisms for ATP synthesis and other vital functions. In visual cells light absorption activates several enzymes in the rod outer segment. Comparative study of the structural organization of the se proteins was necessary to elucidate their functioning as light energy transducers. The complete amino acid sequences of bacteriorhodopsin and rhodopsin were established and their polypeptide chain arrangement in the corresponding membranes was determined. Monoclonal antibodies were used to define the topography of bacteriorhodopsin in the purple membrane. Much more groups of the polypeptide chain were demonstrated to be exposed into the aqueous phase in rhodopsin than in bacteriorhodopsin. The polypeptide chain of rhodopsin traverses the photoreceptor membrane seven times, the $\mathrm{N}^{-}$and $\mathrm{C}-$ terminal parts being located at the opposite sides of the membrane. A general feature for both rhodopsin and bacteriorhodopsin is location of the retinal chromophore binding lysine residue at the C-terminal intramembrane fragment. Data will be presented on the orientation of the retinal and aldimine bond in the membrane.
\end{abstract}

Knowledge of the molecular mechanism of photoreception is a key problem in the study of the visual process as a whole. Rhodopsin, a basic protein component of disk membranes from outer segments of rods from the vertebrate retina, plays a substantial role in photoreception initiating the visual perception. Therefore structure-functional investigations of rhodopsin are essential for understanding physicochemical processes of vision. Of much significance is the mechanism of light energy transformation from the rhodopsin molecule which absorbs light quanta to a cytoplasmic membrane and further to the synaptic ending of the cell, viz. mechanism of photoreception.

Nowadays two possible mechanisms of the process are discussed, each suggests a mediator that regulates the degree of polarization of the plasma membrane.

Cyclic GMP is one of the main mediators whose concentration lowering in cytoplasm, resulted from light-dependent activation of cyclo-GMP-phosphodiesterase, leads to blocking sodium channels. Calcium ions are also considered as probable mediators (ref. 1). A special GTPbinding protein or transducin, a transmitter of the visual signal from rhodopsin to phosphodiesterase and its amplifyer, participates in coupling between photoactivable rhodopsin and phosphodiesterase. Transducin, a peripheral membrane protein, consists of three subunits: $\alpha(39 \mathrm{kDa}), \beta(37 \mathrm{kDa})$, and $\gamma(8 \mathrm{kDa})$ (ref. 2). The major pathways of the model proposed by Fung and Stryer (ref. 2, 3) are summarized as follows. Photolyzed rhodopsin catalyzes the exchange of GTP for GDP bound to the a-subunit of transducin. Photolyzed rhodopsin has a high affinity for transducin-GDP but a low affinity for transducin-GTP. The shift in the binding affinity enables the photolyzed rhodopsin to recycle rapidly and to activate many transducin molecules. The binding of GTP leads to the dissociation of the $\alpha-\beta \gamma$ rhodopsin complex. Since dissociation of the $\alpha$-subunit-GTP complex is much faster than hydrolysis of GTP, the level of the $\alpha$-subunit-GTP complex increases rapidly. The $\alpha$-subunit-GTP complex is an activator of phosphodiesterase. It interacts with phosphodiesterase by removing an inhibitory effect of the $\gamma$-polypeptide on the $\alpha$-and $\beta$-polypeptides of the phosphodiesterase. The enzyme activity is again inhibited when the GTP on the $\alpha$-subunit is hydrolyzed to GDP. The $\beta \gamma$-subunit serves to bring the $\alpha$-subunit back onto rhodopsin then the whole cycle is repeated again.

Until recently rhodopsin was the only known light-transducing protein using retinal as a chromophore. The unexpected discovery, seemingly bearing no direct relation to visual excitation, gave a new impetus to these investigations (ref. 4).

Halophilic microorganisms of the Halobacterium family utilize the solar radiation energy due to the presence of bacteriorhodopsin, a light-driven primary proton translocase. Bacterio- 
rhodopsin is a relatively small protein (about 250 amino acid residues) containing protonated aldimine of the retinal as a prosthetic group. In the cell membrane bacteriorhodopsin is concentrated in patches of ca $0.5 \mu$, called purple membranes. Purple membranes are two-dimensional quasi-crystals formed by hexagonally packed protein trimers, the space between them being filled with lipid molecules. Each working cycle of bacteriorhodopsin induced by the light quantum absorption is accompanied with transfer of at least one proton across the membrane, the retinal aldimine is reversibly deprotonated in the course of this cycle, as judged from spectral data. Bacteriorhodopsin makes up $75 \%$ of the total weight of the purple membrane the remainder being a special set of phospholipids. By electron microscopic and diffraction methods Henderson and Unwin determined the three-dimensional structure of bacteriorhodopsin to a resolution of $7 \AA$ within the membrane plane and about $14 \AA$ perpendicular to the plane (ref. 5). According to these data the bacteriorhodopsin molecule consists of seven roughly parallel segments each spanning the membrane. Unwin and Henderson's three-dimensional model of bacteriorhodopsin left unresolved the question of the actual build up of its active site and arrangement of functional groups in the molecule.

Our interest was focused on chemical and biochemical aspects of this unique membrane protein functioning. No doubt, such studies are necessary not only for elucidation of the mechanism of proton translocation by bacteriorhodopsin but also for understanding the dynamics of functioning of even more complex light energy transducing membrane proteins - visual pigments, halorhodopsin, etc. A recent review from this laboratory (ref. 6) presents data concerning the structural basis for bacteriorhodopsin and rhodopsin function. A model which correlates the bacteriorhodopsin amino acid sequence and three-dimensional structure was elaborated. $\alpha$-Helical rods appeared to have their hydrophobic sites facing the nonpolar lipid moiety, whereas more hydrophilic portions are in the molecule interior.

Comparison of the properties of native bacteriorhodopsin and bacteriorhodopsin with modified dimethylated lysine residues proves that retinal does not change its attachment site (Lys216) during the photocycle, proton translocation and light-dark adaptation. Furthermore, $\varepsilon-$ amino groups of the lysine residue do not play a key role in the coordinated pathway of proton translocation (ref. 7). As judged from fluorescence, UV and CD data the chromophore is located at $9 \AA$ from the membrane surface. That was documented by retinal fluorescence quenching by lanthanide and $\mathrm{Co}^{++}$ions as well as by the analysis of energytransfer between the retinyl or retinyliden moieties and 4-sulfophenylazo group at modified Tyr 64 or $\operatorname{Tyr}\left(\mathrm{NH}_{2}\right)^{64}$ side chain, respectively.

The three-dimensional picture of bacteriorhodopsin at atomic resolution will hopefully emerge from X-ray crystallography data. Leaving this for crystallographers we started a detailed immunological investigation of bacteriorhodopsin which proved to be useful in studying orientation and surface topology of the protein. Khorana et al. used this approach to identify distinct antibody binding sites on the cytoplasmic surface of bacteriorhodopsin (ref. 8) that allowed identification of a peptide loop between $\alpha$-helical segments 3 and 4 and confirmation of the cytoplasmic location of the exposed C-terminal tail. In the course of immunological studies we obtained five hybridomas producing monoclonal antibodies to different membrane exposed parts of the polypeptide chain. Specificity of these antibodies was established using modified derivatives of bacteriorhodopsin and a number of overlapping peptides, derived from enzymatic or chemical cleavages of the protein.

The antigenic determinants are situated on the following exposed parts of bacteriorhodopsin Glul-Glu9 with three N-terminal amino acids; Gly ${ }^{35}-$ Met $^{56}$ including Asp 36 and/or Asp ${ }^{38}$ and $\mathrm{Phe}^{42}$; Phe156-Met 163 with Phe156; Glu194-Leu207 including residue Glu194; Pro200-Leu207. Thus bacteriorhodopsin fragments 4-65 and 156-231 have membrane exposed peptide regions. All the data, experimentally obtained and earlier available, concerning the membrane location of fragments 66-72 and 231-248, evidence that each of the sequences 4-65 and 156-231 traverses the membrane at least twice (Fig. 1).

of even more importance is accessibility of $\mathrm{Glu}^{194}$ to a monoclonal antibody. Upon the study of the chromophore orientation in bacteriorhodopsin by cross-linking using the photosensitive p-diaziridinophenyl analog of retinal, Ser 193 and Glu194 were shown to be the sites of cross-linking with the diaziridine group located at the phenyl ring (ref. 9). Based on this finding a structural model with Glu194 well in the membrane was put forward. Our result, on the contrary, proves that this residue, being a part of an antigenic determinant, should be located on the membrane surface. Obviously, only further studies will establish the real topography of bacteriorhodopsin and its chromophore in the membrane.

Proton translocation and charge movement across the purple membrane were investigated by various techniques. Upon comparing the kinetics of spectral, electrical and pH responses of bacteriorhodopsin induced by a 3 nanosecond laser flash, the correlation was found between formation of the M 412 intermediate as well as proton extrusion and the microsecond stage of electrical potential generation. As to the M 412 decay and proton uptake, they correlate with the millisecond stage of the potential generation. The ratio of micro- and millisecond stages suggests that the outward proton-conducting path is about four times shorter than the inward one. 


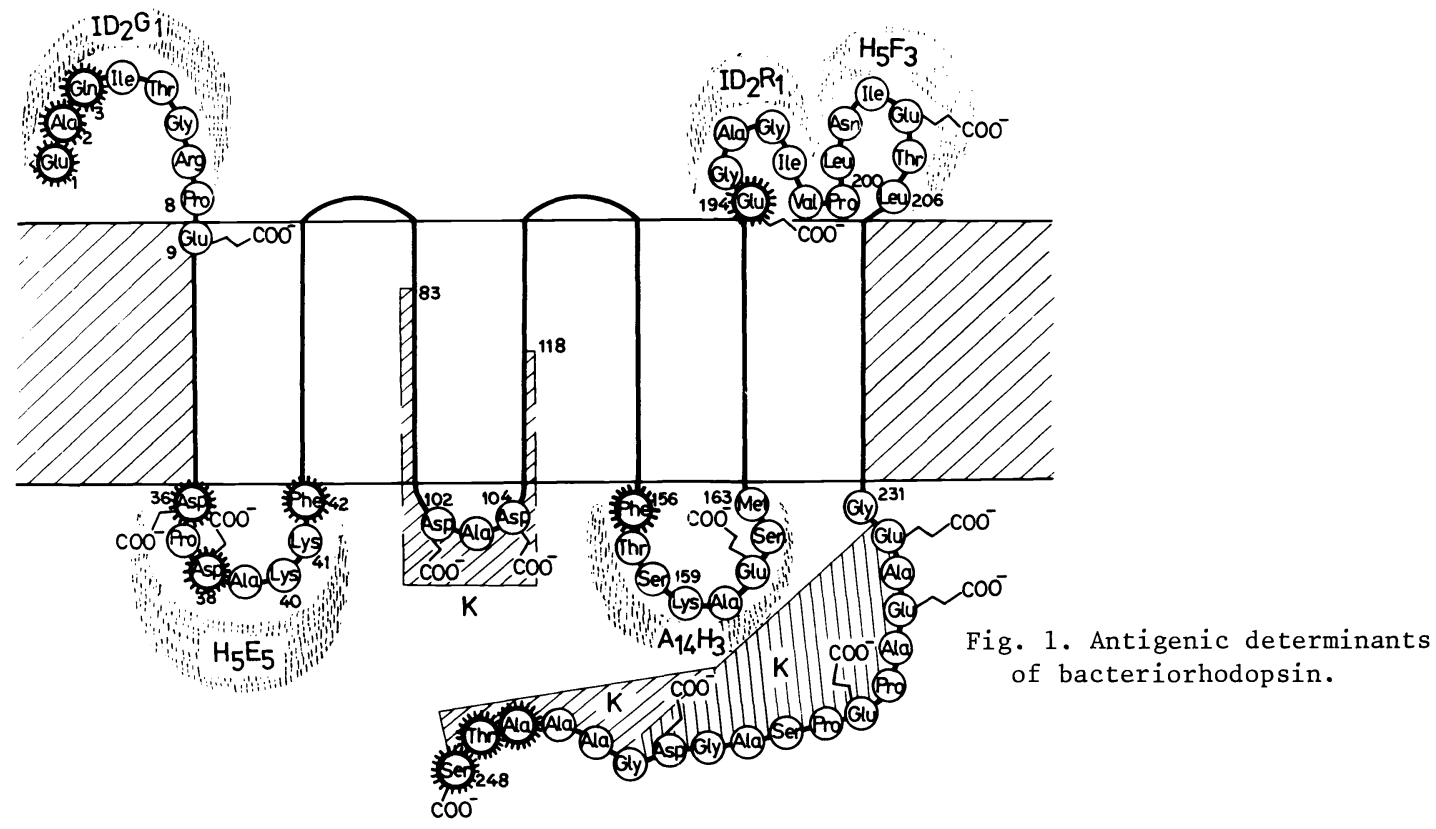

Chemical enzymatic modification of the bacteriorhodopsin protein moiety can give important structure-functional information. As found removal of the C-terminal 17 amino acid residues did not affect efficiency of the proton transport in bacteriorhodopsin (ref. 10). However, according to the latest results the light induced proton release decreases by $50-70 \%$ in such preparations without affecting a photocycle. The data now available confirm our finding that removal of the C-terminal tail influences neither the rate nor the efficiency of the proton transport. An apparent decrease in the efficiency is due to aggregation of the protease treated membrane sheets, the effect being completely prevented by a detergent (ref. 11) (Fig. 2).

Bacteriorhodopsin reconstituted into artificial lipids is a useful model to study many chemical and biochemical aspects of proton translocation in membrane systems, to understand the principles of their denaturation and renaturation. The complete delipidation and reconstitution of the proton pumping activity were achieved in a pioneering work of Khorana (ref. 12, 13). The completely delipidated bacteriorhodopsin was obtained by chromatography of a protein sample solubilized in Triton X 100 on Biogel. The delipidated protein retained its spectral integrity. The vesicles obtained from this preparation by adding different lipids manifested the proton pumping activity. Rather important were the experiments which demonstrated reconstitution of the proton pumping activity from two separate and completely denatured chymotryptic fragments of bacteriorhodopsin (ref. 14). These studies opened the way for purposeful experiments which shed light on the structure-functional relationships in bacteriorhodopsin.

\section{INTACT BACTERIORHODOPSIN}

$\mathrm{H}^{+} / \mathrm{M} 412$

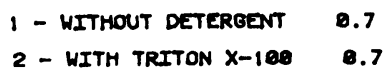

0.7

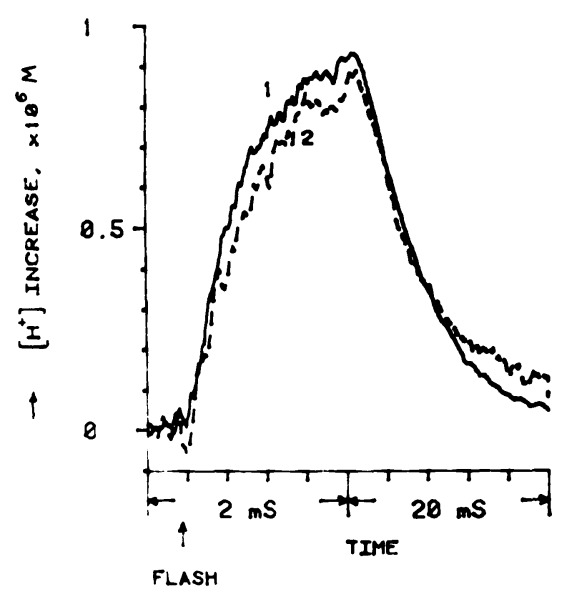

\section{BACTERIORHODOPSIN WITHOUT C-TAIL}

\section{$\mathrm{H}^{+} / \mathrm{M412}$}

$$
\begin{array}{ll}
1 \text { - MITHOUT DETEREENT } & 0.24 \\
2 \text { - MITH TRITON X-100 } & 0.7
\end{array}
$$

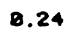

\section{.24}

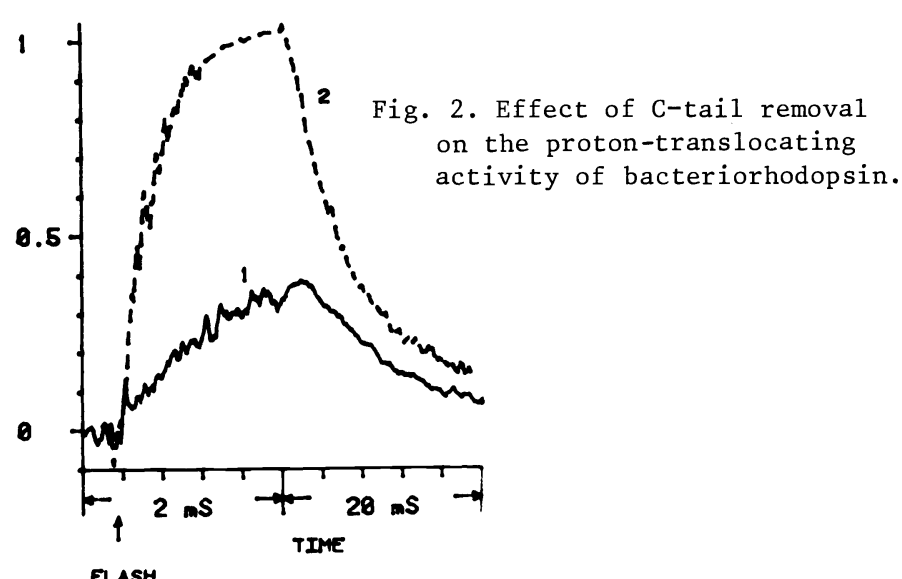


To clarify a role of each bacteriorhodopsin fragment the attempts are made to obtain a hybrid system by exchanging the fragments of bacteriorhodopsin and rhodopsin in reconstitution experiments.

Along with these studies we started an investigation into the genetics of halobacteria searching for new ways to approach the mechanism of the proton translocation.

The structure-functional studies of bacteriorhodopsin pose a number of questions of fundamental importance. Here we would like to know: a) what is the nature of a considerable spectral shift of retinal in bacteriorhodopsin $(380-570 \mathrm{~nm}), \mathrm{b})$ is it possible to prove or disprove the models underlying the active sites of light sensitive proteins, i.e. how valid is the point-charge model, c) are there any connections between the spectral intermediates of pigment bleaching and different stages of proton translocation in bacteriorhodopsin, d) what is the role of membrane-bound and exposed parts of the protein molecule in the pigment function, e) how valid is the conception of proton translocation through the chain of hydrogen bonds and, mostly important, could the proton pumping activity of bacteriorhodopsin be inhibited by replacing the potential "participants" of this pathway by means of site-directed mutagenesis - the technique with many advantages compared to direct chemical modification? Taking into consideration the presence of the 6 th and 7 th $\alpha$-helical rods in functional groups (Fig. 3), namely; Asp212 - the carboxyl group participating in protonation of retinal aldimine, Lys 216 - the retinal attachment site, and two Trp as well as one Tyr residues, we decided to replace these amino acids.

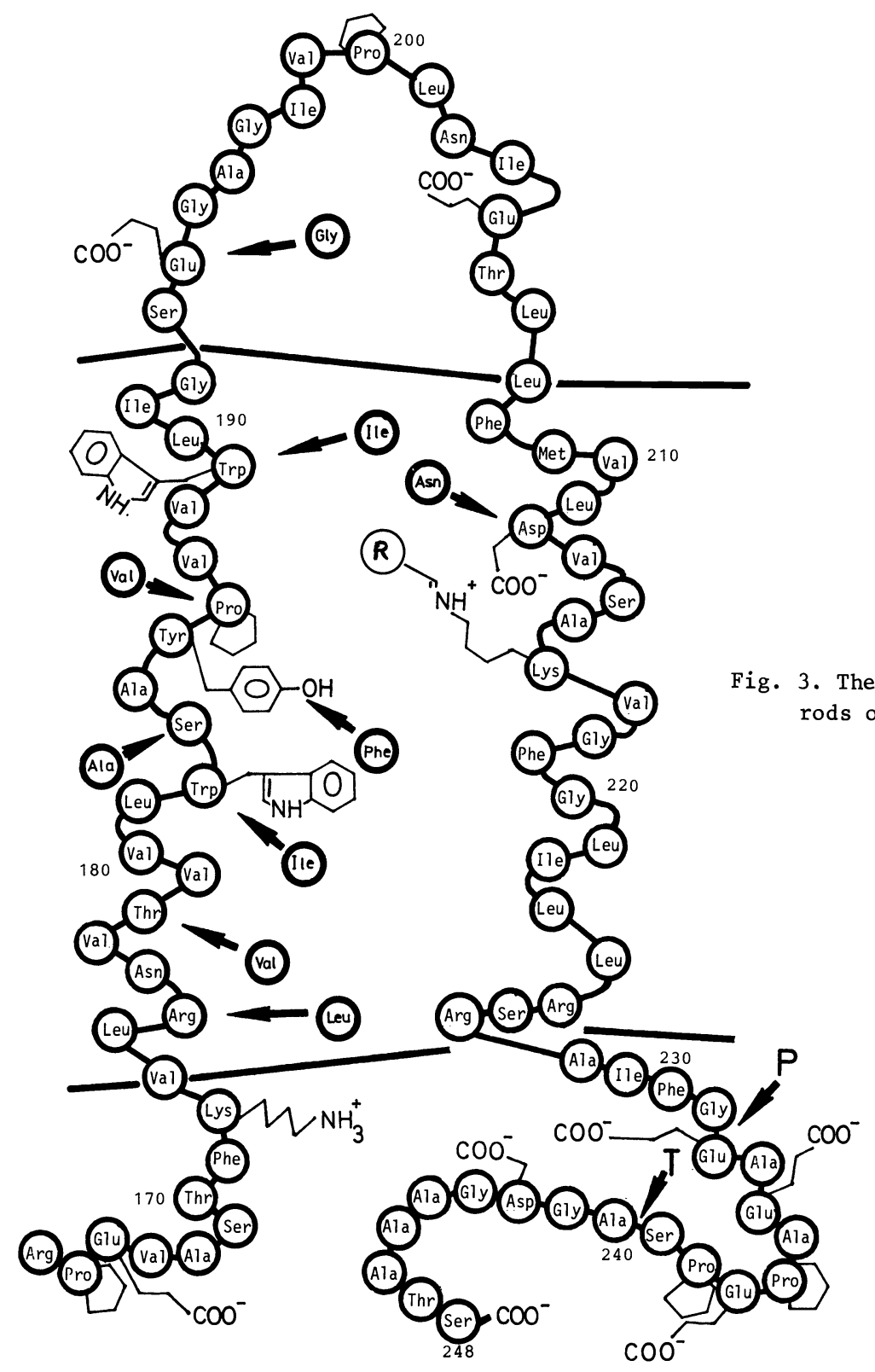


Naturally, all the questions can be solved only by gene manipulation utilizing the recombinant DNA technique; of vital importance here is finding of conditions for high level expression of the bacteriorhodopsin gene. Several recombinant plasmids were constructed to achieve the expression of bacterioopsin in $\mathrm{E}$. coli (Fig. 4). To detect the level of expression we decided to exploit the well known fact that $\beta$-galactosidase of $\mathrm{E}$. coli forms enzymatically functional hybrids if its several N-terminal amino acids are exchanged for some other polypeptide. It is easy to test expression of any foreign protein in E. coli if its C-terminal part is fused to the gene coding for $\beta$-galactosidase with several $N$-terminal codons cut off. Such a hybrid gene placed under promoter control programs synthesis of the hybrid protein possessing galactosidase activity and containing the polypeptide chain of the foreign protein of interest.

The bacterioopsin gene was fused to the lac $\mathrm{Z}$ gene (gene of $\beta$-galactosidase) and this hybrid gene was placed downstream the $\mathrm{E}$. coli tryptophan promoter containing also the ribosome-binding site. This plasmid POG contains the full bacterioopsin gene. It means that this gene encodes an opsin precursor which is 13 amino acid longer than the mature opsin. Judging by $\beta-$ galactosidase activity the level of expression was rather low.
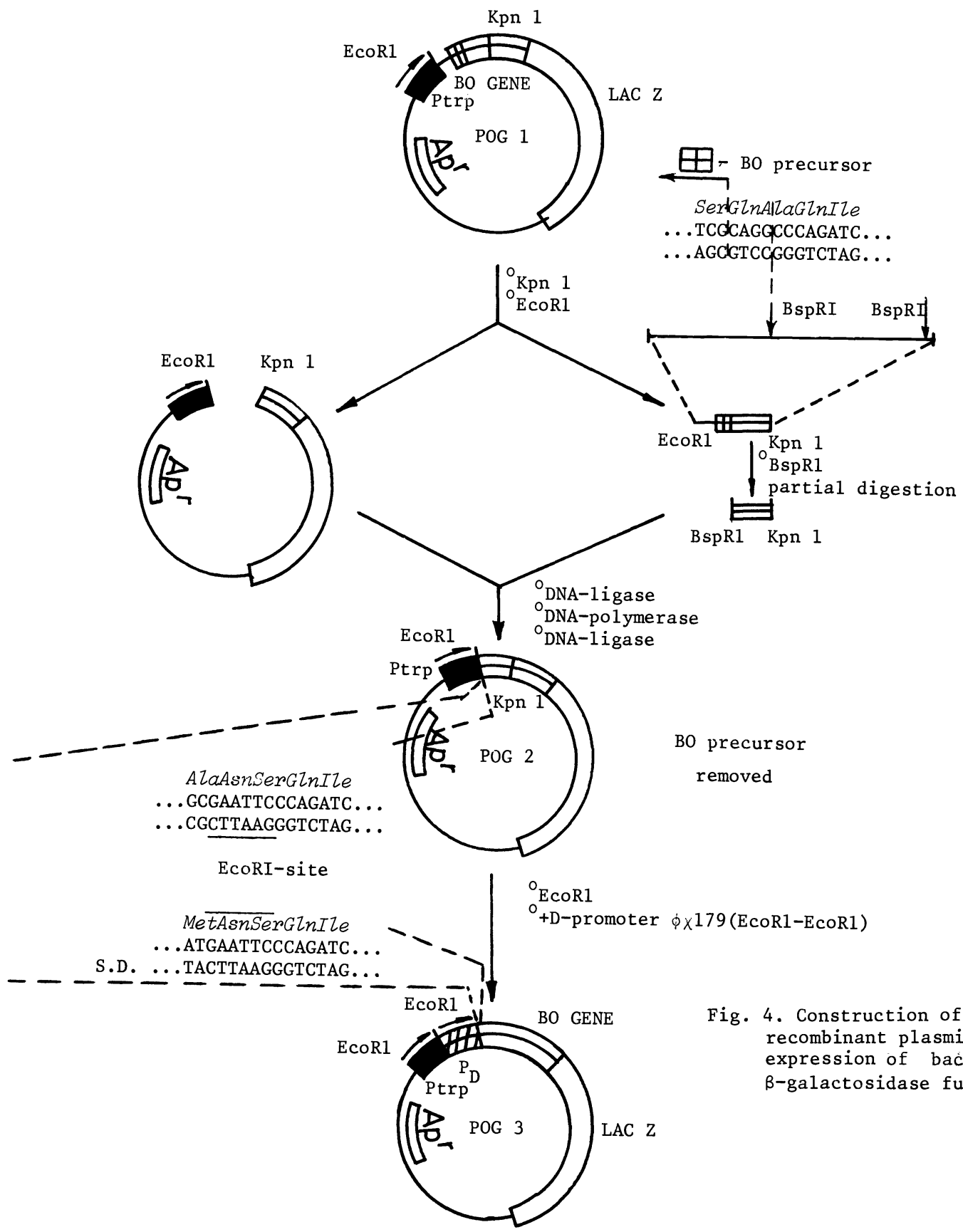

Fig. 4. Construction of the POG 3 recombinant plasmid for the expression of bacterioopsin$\beta$-galactosidase fusion protein. 
We removed the signal peptide and changed the system of expression regulation. The POG 1 plasmid was reconstructed as follows. The precursor region of opsin gene was removed and a strong promoter of phage $\phi \times 179$ containing its own ribosome-binding site and initiating ATG-codon was placed upstream the gene. High galactosidase activity implies a high level of hybrid protein expression (up to $1 \%$ of total cellular protein). The same level of expression was achieved using plasmid pCP3 under the control of the $\lambda_{\mathrm{PL}}$ promoter. This opens wide perspectives for investigation into site-directed mutagenesis of bacterioopsin gene aiming at the production of mutated bacterioopsins and their functional studies. The nearer we are to understanding of the mechanism of bacteriorhodopsin functioning, the more surprising questions arise whose solution requires new efforts and novel approaches.

Now let us see how new ideas and experimental methods created in the rapidly developing studies of bacteriorhodopsin can be applied to its analog - visual pigment rhodopsin.

We determined the complete amino acid sequence of rhodopsin and showed that the polypeptide chain of the protein consists of 348 amino acid residues (ref.6). Related results were obtained recently in the USA (ref. 15) by sequencing a structural gene of bovine rhodopsin. Nucleotide sequence analysis of the cloned DNA provided an intron-exon map of the gene.

According to our data the characteristic feature of the amino acid sequence of rhodopsin is the presence of extended regions of the polypeptide chain made up of nonpolar amino acid residues interrupted with comparatively small sites of polar residues. These hydrophobic extended regions compose the membrane part of the protein molecule.

The primary structure of rhodopsin underlay elucidation of the polypeptide chain arrangement in the membrane. The model building demands the combination of two approaches: a) analysis of the regions of the protein polypeptide chain located in the aqueous phase and accessible to the action of proteolytic enzymes; b) localization of the protein molecule regions containing the least number of polar amino acid residues and capable of spanning the lipid bilayer. Besides, two considerations are taken into account. First, membrane regions of the molecule have the $\alpha$-helical conformation and are situated perpendicularly to the membrane plane; secondary, the $\mathrm{N}-$ and $\mathrm{C}$-terminal regions of rhodopsin are located on opposite sides of the membrane and, consequently, the polypeptide chain of the protein molecule should traverse the membrane uneven number of times. Now let us follow the path of the polypeptide chain in the membrane. Thirty amino acid residues in the $\mathrm{N}$-terminal region of the protein molecule are accessible to the action of various proteolytic enzymes upon the treatment of inside-out photoreceptor disks. Consequently, the $\mathrm{N}$-terminal region of 30 amino acid residues is localized in the intradisk space. The region accessible to the chymotrypsin action was identified on the outer surface of photoreceptor disks (residues Phe146-Arg147). The region of 27 amino acid residues of the polypeptide chain in the $\alpha$-helical conformation can traverse the lipid bilayer of the membrane (to span the entire membrane width 26-30 residues are necessary). Taking this finding into account in the analysis of distribution of polar and nonpolar residues of the polypeptide chain between two regions of proteinolysis (Tyr30-Phe146), we identified three membrane segments separated with two small clusters of hydrophilic residues (regions 62-73 and 92-101). Consequently, region Tyr30-Phe146 of the polypeptide chain traverses the photoreceptor membrane three times. The region accessible to the papain action (Ser ${ }^{186}$-Cys ${ }^{187}$ ) is located on the intradisk membrane surface. Apparently, the region of 40 amino acid residues can span the membrane only once; it contains membrane segment Ile154Ser 176

On the outer surface of photoreceptor disks there is rather extended region Gln 236 -Lys 245 accessible to the action of different enzymes. Since this region and the papain-accessible one are on different membrane surfaces, the polypeptide chain site between them (55 amino acid residues) spans the membrane uneven number of times. This region appeared to contain the only membrane segment (Phe203-Phe228).

The C-terminal region of rhodopsin is situated on the outer disk surface as region $\mathrm{Gln}^{236}$ Lys 245 . The polypeptide chain part connecting these two regions traverses the membrane even number of times. At the study of distribution of polar and nonpolar amino acid residues of this part of the protein molecule ( 75 residues) two membrane segments (Met $253-$ Phe $^{276}$ and Phe ${ }^{283}-$ Met $^{309}$ ) were identified.

Thus seven segments of the polypeptide chain in the $\alpha$-helical conformation, spanning the photoreceptor membrane width, compose the membrane moiety of the rhodopsin molecule (Fig. 5). The $\mathrm{N}-$ and $\mathrm{C}$-terminal regions are the largest sites exposed into the water phase.

There are three functionally important domains in the rhodopsin molecule: retinal-binding site, C-terminal region and polypeptide region connecting the $\mathrm{V}$ and VI membrane segments.

Retinal and rhodopsin have absorption maxima $\sim 380 \mathrm{~nm}$ and $498 \mathrm{~nm}$, respectively, for such considerable bathochromic shift $(120 \mathrm{~nm})$ the charged groups should be incorporated into the retinal binding site. Besides, spectral investigations show that the Trp residue should be 1ocated in the active site of retinal. Lys 296 , responsible for the retinal binding, is located in the VII segment in the middle of the lipid bilayer in accord with data obtained at the study of the energy transfer between the chromophore and terbium ions. It is noteworthy Ala 


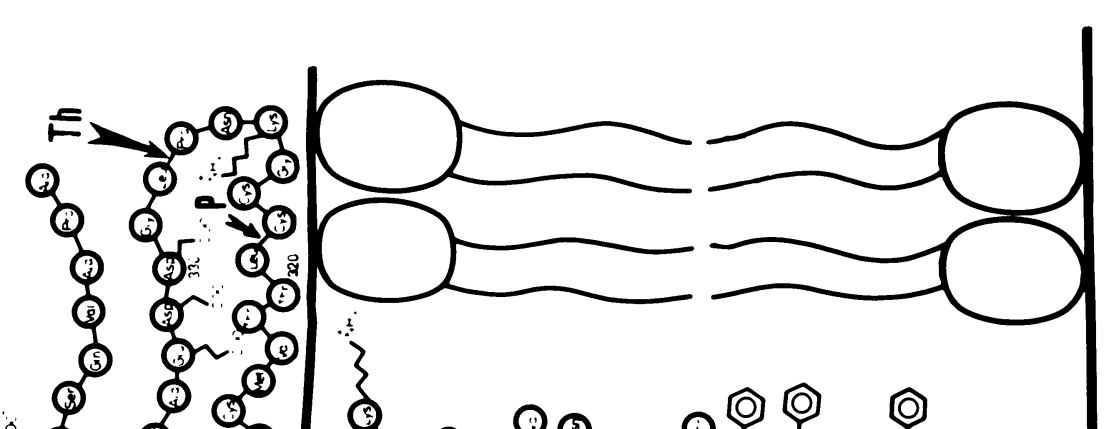

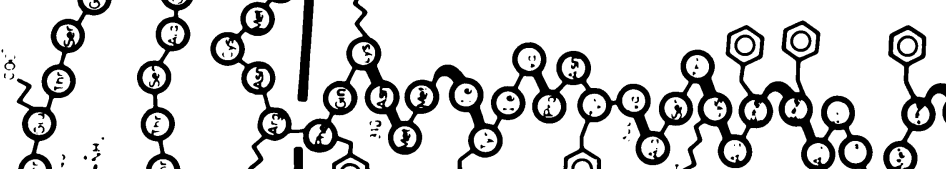
- 0.90 .0308 $=0.100$ 5. . 1010

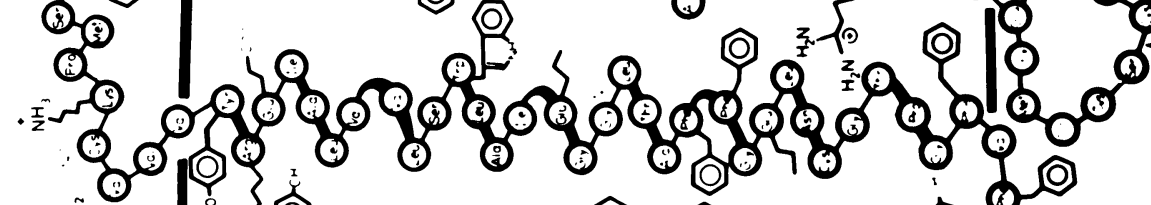
=

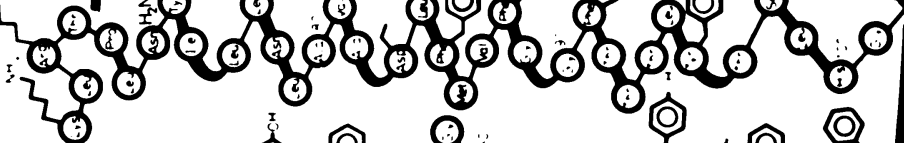
68 $80808 \%$

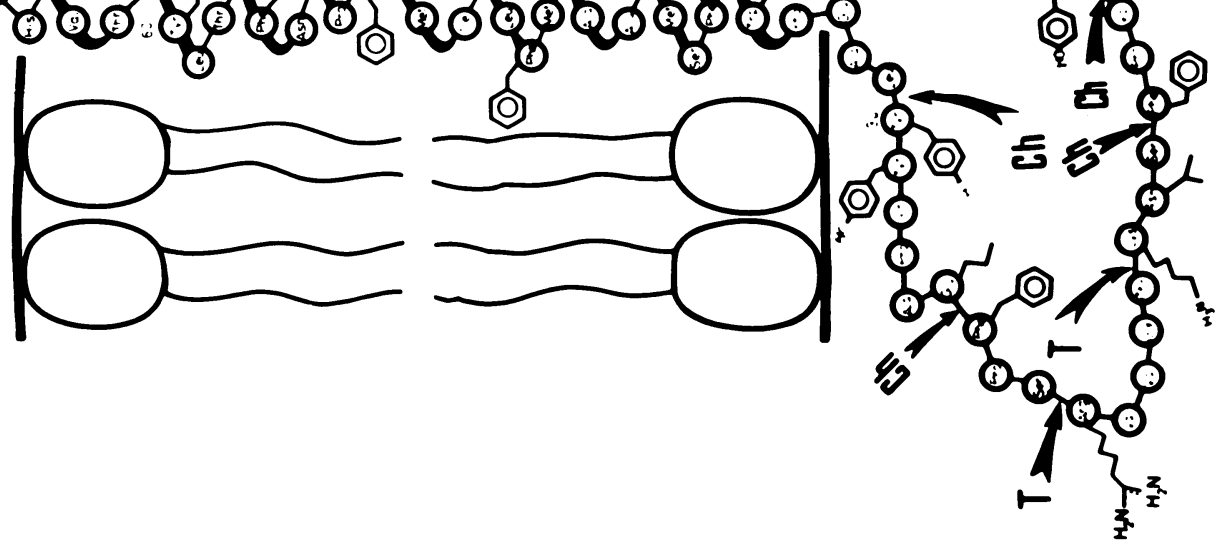


precedes the retinal binding Lys residue in all the studied retinal binding proteins. The absence of the three-dimensional model of the rhodopsin arrangement precludes localization of other amino acid residues in the active site of the protein. As known retinal is located at the $16-23^{\circ}$ angle to the membrane plane, i.e. almost parallelly to its surface. Upon studying various chromophore analogs the length of the retinal binding region was established to be 10.1-10.9 $\AA$. The $\beta$-ionone ring of retinal, or at least its two methyl groups, are extremely important for the prosthetic group binding.

The study of the model compounds shows that bathochromic shift $(120 \mathrm{~nm})$ is possible if a carboxyl group of aspartic of glutamic acids is at the distance of $3 \AA$ from the protonated Schiff base. The second negative charge should be localized within the region of double bond $C(11)-$ $\mathrm{C}(12)$ in the chromophore polyene chain. According to the proposed model three negatively charged amino acid residues Asp ${ }^{83}, \mathrm{Gln}^{113}$ are in the lipid matrix of the photoreceptor membrane, one or two of them can regulate the bathochromic shift of the visual pigment at the interaction with its chromophore. In addition, negatively charged amino acid residues, such as Glu134 or Glu150, located in the vicinity of the membrane surface, can be included in the retinal binding site.

According to the model of the rhodopsin polypeptide chain arrangement, four of five Trp residues (positions 126, 161, 175 and 265) are in the lipid matrix of the photoreceptor membrane. Each of them can be involved in the active site of the protein, however, $\operatorname{Trp} 265$ located in the VI segment in the vicinity of the aldimine bond seems to be the most probable.

The active site of the protein includes the His residue, its imidazole ring can be involved in the proton transfer upon the rhodopsin functioning. In the lipid matrix of the membrane the only residue, His 211 , is situated. The data on the tertiary rhodopsin structure, chemical modification of the protein, application of the photoaffinity retinal analog provide detailed information on the retinal binding region of the visual pigment. A second functionally important rhodopsin domain is the C-terminal protein region exposed into the water phase. This region of the polypeptide chain is phosphorylated that results in inhibition of the action of the photolyzed molecule of the visual pigment (see below). Determination of the primary structure of the C-terminal region revealed seven possible sites of kinase action.

There exist data on participation of the C-terminal domain in formation of its complex with GTPase; except for the finding that cleavage of the 12-membered peptide at the short time thermolysine action (region $\mathrm{Val} 337-\mathrm{Ala}^{348}$ ) does not change the pigment-enzyme binding.

The C-terminal region of rhodopsin contains three of ten Cys residues (positions 316, 322, and 323). Cys 316 is accessible to the action of SH-blocking reagents upon the treatment of the native protein in the photoreceptor membrane, whereas residues Cys 322 and Cys 323 are not modified even after the pigment bleaching. The study of the chemistry of rhodopsin sulfhydryl groups by covalent chromatography on the basis of thiol-disulfide exchange shows that after immobilization of the protein with reduced disulfide bonds and cyanogen bromide cleavage Cterminal fragment (containing residues Cys 322 and Cys323) is covalently bound to the carrier. If disulfide bonds are not reduced, cyanogen bromide fragment forms no covalent bond with the carrier. Being isolated and incubated with dithiotreitol this peptide is capable of immobilization. The data obtained indicate the presence of the disulfide bond between residues Cys 322 and Cys 323 in the C-terminal region of the rhodopsin molecule. The study of the fragment by the resonance Raman spectroscopy in the region of the $S$ - S valence vibrations supports this. That is the first case of discovering the disulfide bond between the adjucent Cys residues in the naturally occurring sample, up to now such a bond has been found only in synthetic peptides.

Though the disulfide bond does not change upon the rhodopsin bleaching, its participation in the thiol-disulfide exchange when forming the pigment-transducin complex is possible, as, for example, the receptor-insulin complex. Modification of transducin with SH-blocking reagents precludes formation of the photolyzed pigment-enzyme complex (the $\alpha$-subunit of transducin is modified).

The third functionally important domain is the polypeptide chain region connecting the $\mathrm{V}$ and VI membrane segments. Apparently, it participates in complex formation with transducin, since at the thermolysin cleavage of this region into two membrane-bound fragments the photoreceptor disks considerably decrease its ability to the enzyme binding. If so, considerable homology in this region among rhodopsin from different sources should be observed. However, careful analysis of sequence data on bovine, human, drosophila rhodopsins shows no homology. Thus this region seems to be less, if not at all essential for binding ampliphier proteins. At the same time in the sequence of octopus rhodopsin, now we study, there is certain homology in the second cytoplasmic peptide loop.

0
D
Bet-Ile-Ser-Ile-Asp-Arg-Tyr-Asn-Val-Ile-Gly-Arg-Pro-Met
Met-Ile-Ser-Leu-Asp-Arg-Tyr-Gln-Va1-Ile-Val-Lys-Gly-Met
Val-Leu-Ala-Ile-Glu-Arg-Tyr-Val-Val-Val-Cys-Lys-Pro-Met

$$
\begin{aligned}
& \text { O - octopus, } \\
& \text { D - drosophila, } \\
& \text { B - bovine }
\end{aligned}
$$


Close similarity in this region may indicate its importance for binding the enzymes involved in signal transduction.

As mentioned above the C-terminal region of rhodopsin contains seven potential sites for the kinase action. However, 9 moles of $\mathrm{Pi}$ can be included into I mole of the protein upon its bleaching. One more site of the kinase action is probably located on the polypeptide chain between the $\mathrm{V}$ and VI membrane segments containing three possible sites of the enzyme action, Ser240, $\mathrm{Thr}^{242}$, and $\mathrm{Thr}^{243}$. If so, incorporation of two phosphate groups in this region after the pigment illumination may regulate the complex formation of photolyzed rhodopsin with GTPase.

Site-derected mutagenesis should hopefully enable us to find out functional roles for these and other parts of rhodopsins.

Function mechanisms of proteins, which participate in the transmission and amplification of the visual signal, in many respects are similar to those for the adenylate cyclase system transferring a hormone signal. The system also consists of three protein components associated with the membrane: a hormone receptor, a catalytic subunit, and a GTP-binding regulatory protein coupling the catalytic subunit and receptor, usually called $\mathrm{N}$ or $\mathrm{G}$ protein. There exist two types of $G$ proteins: stimulatory and inhibitory. Activation of the hormone receptor similarly to the visual system promotes the GDP-GTP exchange at the G protein. The protein and receptor form a stable complex in the presence of the hormone. The GTP binding triggers dissociation of the complex. The stimulatory G protein in the GTP-form activates a catalytic component, that increases cyclo-AMP concentration. GTP hydrolysis also transforms a catalytic component into an inactive form. Certain protein components in both the systems turned out to be interchangeable. Thus photolyzed rhodopsin is capable of GTP-dependent activation of adenylate cyclase. The complex of transducin with guanosine-5'| $\beta, \gamma \mid$ imidotriphosphate acts in the same way (ref. 16).

Especially close homology exists between transducin and the inhibitory G protein. These proteins have an identical subunit composition, some of their subunits are interchangeable, they have similar amino acid compositions and peptide maps (ref. 17).

There is a series of proteins with similar structure-functional properties as transducin and $G$ protein of the adenylate cyclase system which bind guanyl nucleotides. Here belong ribosomal elongation factors $\mathrm{Tu}$ and $\mathrm{G}$, tubulins as well as human bladder protein P21 and its oncogenic variants (ref. 18).

We decided to study the proteins involved in visual and hormone signal transmission due to significance and universal character of the regulatory system using guanyl nucleotides.

We started with elucidation of the transducin amino acid sequence. Two methods underlay separation of its subunits: electrophoresis in the acetate cellulose block under denaturing conditions and HPLC. Electrophoresis is highly productive; a few dozens of milligrams of transducin can be separated simultaneously on the block - 30x40, $5 \mathrm{~mm}$ in width. However, because of close mobility pure $\alpha$ - and $\gamma$-subunits cannot be often obtained. HPLC on the column with reversed phase Silasorb-Phenyl turned out to be mostly effective for pure $\alpha, \gamma$ subunits. The subunits were eluted in acetonitrile gradient in $0.1 \%$ trifluoroacetic acid.

The amino acid composition was determined for every subunit. Despite the close molecular weights, the amino acid compositions of the $\alpha$ - and $\beta$-subunits have many differences. In particular, the content of glutamic acid, methionine, and arginine residues differs. The $\gamma$-subunit is characterized by the absence of alanine, histidine, and triptophan residues. The amino acid sequence of the transducin $\gamma$-subunit was established as follows. $34 \mathrm{~N}$-terminal amino acid residues were determined by the automated sequencing. Use was made of the block technique to define the complete amino acid sequence of the $\gamma$-subunit. To fragmentize the poly-peptide chain of the $\gamma$-subunit hydrolyses with Staphylococcus aureus protease and chymotriposin as well as cyanogen bromide cleavage were used.

By HPLC on the reversed phase Nucleosil C18, 9 and 12 peptides, respectively, were isolated from enzymatic hydrolyzates; $\mathrm{N}-$ and $\mathrm{C}-$ terminal cyanogen bromide fragments were also obtained, as a result a complete amino acid sequence of the $\gamma$-subunit was determined (ref. 19) (Fig. 6).

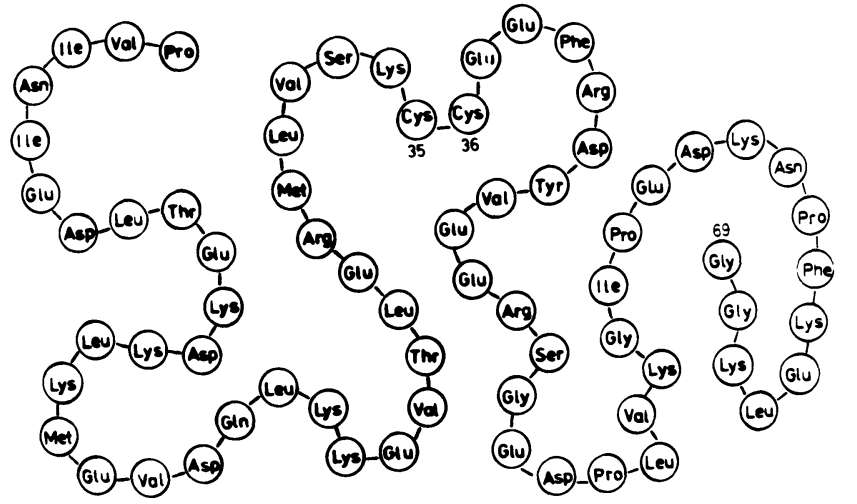

Fig. 6. $\gamma$-Subunit of GTP-binding protein. 
The clostripain hydrolyzate of the $\gamma$-subunit was analyzed on a ZAB(VG) mass spectrometer by using ionization with fast atom bombarding. The obtained mass-spectrum mostly consisted of molecular ions of clostripain peptides. Their molecular masses coincided with those expected, that confirmed the structure obtained.

The transducin $\gamma$-subunit consists of 69 amino acid residues, $\mathrm{M}_{\mathrm{r}} \sim 8008$. The peculiarity of the $\gamma$-subunit is the presence of two neighbouring cysteine residues in positions 35 and 36 , probably, linked with a disulfide bond. Perhaps such an unusual bond plays an essential part in supporting specific conformation of the protein. The $\gamma$-subunit seems to have the $\beta$-turn formed by an ionic pair of 1 ysine-34 and glutamic acid-37 along with cysteine residues.

Not long ago Khorana and Hurley (ref. 20, 21) established the nucleotide sequence of the structural gene of the $\gamma$-subunit. The amino acid sequence deduced coincides with our structure. However, the sequence determined by Khorana and Hurley contains an additional residue of methionine in the $\mathrm{N}$-terminus, and in the C-terminus - four additional residues of cysteine, valine, isoleucine, and serine, which are evidently cleaved off at the protein processing.

At present we are engaged in investigation of the primary structure of the transducin large $\alpha$ - and $\beta$-subunits, simultaneously the work on isolation of the protein structural gene is performed. Freshly isolated protein preparations were found out to be easily hydrolyzed with both proteolytic enzymes and cyanogen bromide. When stored the $\alpha-$ and $\beta$-subunits of transducin are quickly denatured and become poorly soluble even in $70 \%$ formic acid or in $6 \mathrm{M}$ urea.

For cyanogen bromide cleavage the most suitable is the mixture of the $\alpha$ - and $\beta$-subunits obtained by dialysis against ammonia water of the fresh transducin preparation solubilized in buffer with $8 \mathrm{M}$ guanidine hydrochloride. The $\alpha$ - and $\beta$-subunits precipitated, and the $\gamma$-subunit remained in solution. The residue obtained was centrifuged, dissolved in formic acid and cleaved with cyanogen bromide. The primary fractionation of cyanogen bromide peptides by gel filtration on Sephadex G-50 equilibrated with $10 \%$ formic acid yielded 7 fractions.

Further separation was performed by HPLC on reversed phase Nucleosil C18 column in $0.1 \%$ trifluoroacetic acid, peptides were eluted in acetonitrile gradient. In total 19 peptides covering $~ 95 \%$ of the amino acid sequence of transducin $\alpha-$ and $\beta$-subunits were isolated from cyanogen bromide hydrolyzate. The $\alpha$ - and $\beta$-subunits of transducin were also cleaved with trypsin and protease from Staphylococcus aureus. Nucleotide probes, used for isolation of transducin structural genes from cDNA library prepared from bovine retinal RNA were synthesized on the basis of the peptide structure (Fig. 7).

Gln-Asn-Va1-Lys-Phe-Va1-Phe-Asp-Ala

$$
\begin{aligned}
& \text { 1. } \quad 3^{\prime}-\mathrm{GT}_{\mathrm{C}}^{\mathrm{T}} \mathrm{TT}_{\mathrm{G}}^{\mathrm{A}} \mathrm{CAN} \mathrm{TT}_{\mathrm{C}}^{\mathrm{T}} \mathrm{AA}_{\mathrm{G}}^{\mathrm{A}} \mathrm{CA}-5^{\prime} \\
& \text { 2. } \quad 3^{\prime}-\mathrm{TT}_{\mathrm{C}}^{\mathrm{T}} \mathrm{AA}_{\mathrm{G}}^{\mathrm{A}} \mathrm{CAN} \mathrm{AA}_{G}^{\mathrm{A}} \mathrm{CT}_{\mathrm{G}}^{\mathrm{A}} \mathrm{CG}-5^{\prime} \\
& \text { 3. Glu-Asn-Leu-Lys-Asn-Cys } \\
& 3^{\prime}-\mathrm{CT}_{\mathrm{C}}^{\mathrm{T}} \mathrm{TT}_{\mathrm{G}}^{\mathrm{A}} \mathrm{AA}_{\mathrm{C}}^{\mathrm{T}} \mathrm{TT}_{\mathrm{C}}^{\mathrm{T}} \mathrm{CT}_{\mathrm{G}}^{\mathrm{A}} \mathrm{AC}-5^{\prime}
\end{aligned}
$$

Fig. 7. Synthetic oligodeoxyribonucleotide probes used to isolate structural genes.

First two probes are of interest because they contain overlapping sequences. In our opinion such set of probes will help us find nonspecific hybridization at cloning of gene libraries, stipulated by the multivariant structure of the probes. The third probe was synthesized on the basis of the peptide structure of the transducin $\alpha$-subunit containing cysteine residue which according to Gilman is subjected to ADP-ribosylation when treating transducin with the Pertusis toxin. As mentioned above transducin has a considerable structure-functional homology with the inhibitory $G$ protein of the adenylate cyclase system. In particular, the protein is also ADP-ribosylated with the Pertusis toxin. We found the last probe to be well hybridized with poly-A (+) fractions of RNA, isolated both from the retina and from brain as well as cerebellum. Since brain and cerebellum possibly do not contain RNA of transducin subunits, the given probe was presumably hybridized with RNA of the $\alpha$-subunit of one of the $G$ proteins of the adenylate cyclase system, and can be used to isolate a relative gene.

Recently Numa (ref. 22) and Lochrie (ref. 23) almost simultaneously published the nucleotide sequence of the cDNA coding for the transducin $\alpha$-subunit. The deduced amino acid 
a)

$\begin{array}{ll}\text { Met-G1y-Ala-G1y-Ala-Ser-Ala-Glu-Glu-Lys-His- } & \text { Ser-Arg } \\ \text { AIIG GGG GCT GGG GCC AGC GCT GAG GAG AAG CAC } & \text { TCA AGG } \\ \text { Met-G1y-Ser-G1y-Ala-Ser-Ala-Glu-Asp-Lys Glu-Leu-Ala-Lys-Arg-Ser-Lys }\end{array}$

b)

$\begin{array}{cccc}1 & 2 & 5 & 10 \\ \text { (Ac)-Met-Ala-Gly-Ala-Ser-Ala-Glu-Glu-Lys-His- }\end{array}$

cyanogen bromide peptide

Fig. 8. N-terminal amino acid sequence of transducin $\alpha$-subunit determined:

a) by Sh.Numa et al. b) S.Hurley et al. c) in my laboratory.

sequences of the $\alpha$-subunit differ considerably (of 350 amino acid residues 69 are unlike). The sequence determined in our laboratory coincides with that found by Numa and covers $\sim 50 \%$ of the $\alpha$-subunit polypeptide chain.

It seems that cDNA isolated by Lochrie et al. corresponds to the gene for some GTP-binding protein homologous to transducin, but not to the gene for the transducin $\alpha$-subunit.

However, there is one difference between our results and those by Numa. We isolated a cyanogen bromide peptide having an alanine residue as the N-terminal amino acid residue (its structure agrees with the sequence 3-49 determined by Numa) (Fig. 8). A specificity of the cyanogen bromide cleavage shows a methionine residue to be located in front of the alanine-3 residue in the polypeptide chain of the $\alpha$-subunit. The sequence Met-Gly precedes alanine according to the structure by Numa. Probably the error took place here because thymidine was missed between guanine-5 and guanine-6. Perhaps that resulted from compression; then the missed thymidine was placed between adenine-1 and guanine-2. After thymidine insertion the GTG triplet was formed which is known to be a second initiating codon, coding for the methionine residue at initiation. The $\alpha$-subunit $\mathrm{N}$-terminal amino group was blocked probably as a result of acetylation.

To study an antigen structure of the transducin molecule and to elucidate the protein function mechanism and the role of its individual subunits, monoclonal antibodies were produced. The native transducin served as an antigen in all hybridizations, for immunization mice of $\mathrm{Ba} 1 \mathrm{~b} / \mathrm{c}$ or $\mathrm{C} 57-\mathrm{B} 1 \mathrm{ack} / 6$ lines were used, hybridization of immune splenocytes was performed with the cells of myeloma Ag8653x63. Sharply positive clones were produced as ascite. Al1 the antibodies were of jik type.

For their purification affinity chromatography on protein A-Sepharose or ion exchange chromatography on DEAE-cellulose were applied.

The specificity of the obtained monoclonal antibodies in relation to the transducin subunits was studied by immunoelectroblotting (Table). The results showed, after electrophoresis under nondenaturing conditions two monoclonal antibodies, A3G7 and A3C10, were specifically bound to the $\alpha$-subunit, A3E4 and 3B3 interacted with the complex $\beta \gamma$, monoclonal antibodies IC3 do not show a clear interaction. According to immunoelectroblotting after electrophoresis in PAAG+SDS gradient, A3G7 and A3C10 were bound to the $\alpha$-subunit, A3E4 - to the $\beta$-subunit, 3B3 and IC3 do not interact with the denatured protein.

Interaction of monoclonal antibodies with isolated subunits was studied by immuno-enzymatic analysis. The $\alpha, \gamma$-subunits and the $\beta \gamma$ complex were used as antigens. A3G7 and A3G10 were shown

TABLE. Specificity of transducin monoclonal antibodies

\begin{tabular}{|c|c|c|c|c|c|}
\hline & 3В3 & $1 \mathrm{C} 3$ & $\mathrm{~A}_{3} \mathrm{G}_{7}$ & $\mathrm{~A}_{3} \mathrm{E}_{4}$ & $\mathrm{~A}_{3} \mathrm{C}_{10}$ \\
\hline $\begin{array}{l}\text { Immunoelectroblotting } \\
\text { under nondenaturing } \\
\text { conditions }\end{array}$ & $\mathrm{Br}$ & $\beta \gamma+\alpha$ & $\alpha$ & $B \gamma$ & $\alpha$ \\
\hline $\begin{array}{l}\text { Immunoelectroblotting in } \\
\text { the presence of SDS }\end{array}$ & - & - & $\alpha$ & $\gamma$ & $\alpha$ \\
\hline Immuno-enzymatic analysis & $\gamma$ & native & $\alpha$ & $\gamma$ & $\alpha$ \\
\hline $\begin{array}{l}\text { Influence on GTPase } \\
\text { activity }\end{array}$ & - & transducin & $\begin{array}{c}100 \% \\
\text { inhibition }\end{array}$ & & $\begin{array}{c}80 \% \\
\text { inhibition }\end{array}$ \\
\hline
\end{tabular}


to be directed against $\alpha$, A3E4 and 3B3 - against $\gamma$, and IC3 interacts neither with isolated subunits nor with the complex, however, it is bound to the native transducin.

Thus A3G7, A3C10 and A3E4 are directed against linear determinants of the $\alpha-$ and $\gamma$-subunits, 3B3 recognizes the determinant of nonlinear type present only in the native $\gamma$-subunit. As to IC3 they, apparently, recognize a complex topographic determinant, formed with all the subunits of transducin that provides use of IC3 to determine the efficacy of the transducin reconstruction from the subunits.

We started investigating the influence of obtained monoclonal antibodies on the transducin molecule function. Monoclonal antibody against $\alpha$ was shown to suppress GTPase activity of transducin almost completely. All the five monoclonal antibodies interact with the native transducin, so they recognize antigen determinants located on the protein surface and, in principle, can catch the molecule regions responsible for interaction of transducin with other components at transduction of the visual impuls, i.e. with rhodopsin and phosphodiesterase. Influence of monoclonal antibodies on transducin interaction with the given proteins is under study. Its interaction with antibodies $\mathrm{A} 3 \mathrm{C} 7$ and $\mathrm{A} 3 \mathrm{C} 10$ suppressing GTPase activity is of special interest. We are eager to find out whether the antibodies are directly bound to the enzyme catalytic site or it prevents formation of the specific complex of transducin with photolyzed rhodopsin leading to the protein activation. Important information on its function is received by localization of antigen determinant of transducin recognized with these antibodies.

There are still many questions to be answered and we hope a comparative study of bacteriorhodopsin and rhodopsin, as well as other proteins participating in transfer and amplification of visual signals may help elucidate mechanisms of light transduction.

\section{REFERENCES}

1. R. Uhe, E.W. Abrahamson, Chem. Rev., 81, 291-312 (1981).

2. L. Stryer, J.B. Hurley, K. -K. Fung, Trends in Biochem. Sci., 6, 245-247 (1981).

3. B.K.K. Fung, J. Biol. Chem., 258, 10495-10502 (1983).

4. D. Oesterhelt, W. Stoeckenius, Nature (New Biol.) 233, 149-556 (1971).

5. R. Henderson, P.N.T. Unwin, Nature 257, 28-32 (1975).

6. Yu.A. Ovchinnikov, FEBS Lett., 148, 179-191 (1980).

7. N.G. Abdulaev, N.A. Dencher, A.E. Dergachev, A. Fahr, A.V. Kiselev, Biophys. Struct. Mech., 10, 211-227 (1984).

8. R. Kimura, T.L. Mason, H.G. Khorana, J. Biol. Chem, 257, 2859-2867 (1982).

9. K.S. Huang, R. Radhakrishnam, H.G. Khorana, J. Biol. Chem., 257, 13616-13623 (1982).

10. N.G. Abdulaev, M.Yu. Feigina, A.V. Kiselev, Yu.A. Ovchinnikov, L.A. Drachev, A.D. Kaulen, L.V. Khitrina, V.P. Skulachev, FEBS Lett., 90, 190-194 (1978).

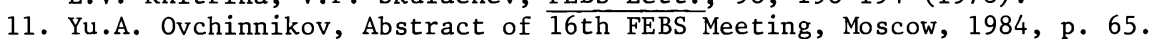

12. E. London, H.G. Khorana, J. Bio1. Chem., 257, 7003-7011 (1982).

13. K.S. Huang, H. Bayley, M.J. Liao, E. London, H.G. Khorana, J. Biol. Chem., 256, 3802-3809 (1981).

14. M.-J. Liao, E. London, H.G. Khorana, J. Biol. Chem., 258, 9949-9955 (1983).

15. J. Nathans, D. Hoggnes, Ce11 34, 807-814 (1984).

16. M.W. Bitensky, M.A. Wheeler, M. Rasenick, A. Yamazaki, P.J. Stein, K.R. Halliday, G.L. Wheeler, Proc. Natl. Acad. Sci. USA 79, 3408-3412 (1982).

17. D.R. Manning, A.G. Gilman, J. Biol. Chem., 258, 7059-7063 (1983).

18. R. Leberman, U. Ehner, EMBO Journal 3, 339-341 (1984).

19. Yu.A. Ovchinnikov, V.M. Lipkin, T.M. Shuvaeva, A.P. Bogachuk, V.V. Shemyakin, FEBS Lett., $179,107-110$ (1985).

20. K. Yatsunami, B.V. Pandya, D.D. Oprian, H.G. Khorana, Proc. Natl. Acad. Sci. USA 82 , 1936-1940 (1985).

21. J.B. Hurley, H.K.W. Fong, D.B. Teplow, W.J. Dreyer, M.I. Simon, Proc. Nat1. Acad. Sci. USA 81, 6948-6952 (1984).

22. T. Tanabe, T. Nukada, Y. Nishikawa, K. Sugimoto, H. Suzuli, H. Takakushi, M. Noda, T. Haga, A. Ichiyama, K. Kaugawa, N. Minamino, H. Matsuo, Sh. Numa, Nature 315, 242-245 (1985).

23. M.A. Lochrie, S.B. Hurley, M.I. Simon, Science, 228, 96-99 (1985). 